\title{
Es wird wieder Zeit für Osler: «Osler's Ten»
}

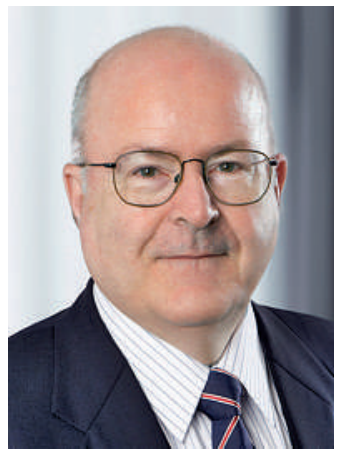

Werner Bauer
Manche Leser werden diesen Titel etwas gereizt zur Kenntnis nehmen: Fällt dem Autor dieser Spalten wirklich keine andere Quelle für seine Texte mehr ein? Einzelne von ihnen werden eher amüsiert vermuten, dass Osler endgültig zum Steckenpferd des schrullig gewordenen Verfassers geworden ist, und auf ein gelegentliches Umsatteln dieses Steckenpferds hoffen. Einige werden sich aber vielleicht doch fragen, welche Bewandtnis es mit «Osler's Ten» haben könnte.

Der Leitartikel des Bulletins 1/12 der Schweizerischen Akademie der Medizinischen Wissenschaften SAMW (M. Louis-Courvoisier) trägt den Titel «Geisteswissenschaften im Medizinstudium: ein Plädoyer für flexibles Denken». Mit dem Hinweis darauf, dass die Medical Humanities neben dem Fachwissen entscheidend zur Entwicklung einer Arztpersönlichkeit (Arzt-Sein) beitragen können, wird der bewusste und vermehrte Einbezug von Literatur, visuellen Künsten, Human- und Sozialwissenschaften in die ärztliche Bildung gefordert. Auch wenn die Lernzielkataloge schon übervoll sind, scheint in den USA und jetzt auch in Europa die Erkenntnis Boden zu gewinnen, dass die Geisteswissenschaften Aspekte des Denkens vermitteln können, die bei der Betreuung von Patienten und bei anderen beruflichen Aufgaben in der Forschung und im Gesundheitswesen hilfreich und wichtig sind. Der Präsident der SAMW, Peter Meier-Abt, formuliert es in seinem Editorial so: «Grenzfragen der modernen Medizin und Biologie können ohne Einbindung von Sozial- und Geisteswissenschaften nicht menschengerecht und gesellschaftsfähig bewältigt werden.»

Auch das Expertenteam, das für die Akkreditierung der Medizinischen Fakultät Bern verantwortlich war, hat im Rahmen seiner Schlusspräsentation eine äusserst positive Beurteilung abgegeben, im Sinne eines Denkanstosses aber auch die Frage aufgeworfen, ob den Studenten nicht wieder vermehrt die Gelegenheit zu Kontakten mit den Geisteswis- accomplished in the course of a year. I have put down a list of ten books which you may make close friends. There are many others; studied carefully in your student days these will help in the inner education of which I speak (Aequanimitas).»

Die zehn ganz unterschiedlichen Werke, die Osler als «bedside library for medical students» ausgewählt hat, sind:

- Plutarch: Vitae parallelae

- Thomas Browne: Religio Medici

- William Shakespeare:

Othello, Sommernachtstraum, Hamlet, Sonette

- Michel de Montaigne: Essays

- Marc Aurel: Meditationen

- Epiktet: Werke

- Miguel de Cervantes: Don Quijote

- Ralph Waldo Emerson: Essays and Lectures

- Oliver Wendell Holmes:

The autocrat of the breakfast-table

- Altes und Neues Testament

Es ging Osler nicht um eine «Bestseller»-Liste für Medizinstudenten, denn er erwähnte in seinen Schriften und Referaten noch eine ganze Reihe von anderen Autoren, die ihn auch beeindruckt haben (von Charles Dickens über Pascal, Voltaire und Ibsen bis zu Avicenna).

Entscheidend war für ihn die Überzeugung, dass die Lektüre wichtiger Werke aus der Weltliteratur das Spektrum des Denkens junger Ärzte entscheidend erweitern kann. Kurz und prägnant hat er formuliert: «The practitioner needs culture as well as learning.»

Von Osler in die Gegenwart: Was ich mir wünsche, sind möglichst viele zukünftige und junge Ärztinnen und Ärzte mit Interesse an der Literatur, an der Sprache als Grundlage des Denkens und Instrument des Heilens, an den bildenden Künsten, an Philosophie und anderen Humanwisssenschaften. Was ich mir zudem wünsche, sind Verantwortliche in Aus- und Weiterbildung, die sich eine Art «Liste der Zehn» (es dürfen auch mehr sein) zusammengestellt haben und hie und da die Zeit finden, mit ihren Studenten oder Assistenzärzten über Gedanken zu diskutieren, die ihnen aus der Lektüre zugeflogen sind. Falls der eine Leser oder die andere Leserin Vorschläge für eine Liste «Osler's Ten 2012» machen möchte, würden mich diese sehr interessieren.

Werner Bauer *

(n)
\footnotetext{
senschaften gegeben werden könnte.

Und damit sind wir wieder bei Sir William Osler und seinen verblüffend aktuellen Ansichten:

«Well filled though the day be with appointed tasks, to make the best possible use of your one or of your ten talents, rest not satisfied with this professional training, but try to get the education, if not of a scholar, at least of a gentleman. Before going to sleep read for half an hour, and in the morning have a book open on your dressing table. You will be surprised to find how much can be

Facharzt für Innere Medizin Instituts für ä Past-President der European Federation of Internal Medicine EFIM.
} 